\title{
Le changement d'échelle du commerce équitable : une réalité empirique
}

\author{
Analyse localisée de l'évolution de deux associations en Bourgogne
}

\author{
Matthieu Gateau \\ Université de Bourgogne \\ Centre G. Chevrier UMR CNRS 5605 \\ Bureau 263, Pôle AAFE \\ Esplanade Erasme \\ 21000 Dijon \\ France \\ <matthieu.gateau@u-bourgogne.fr>
}

\begin{abstract}
Résumé
En France, depuis le début des années 2000, le commerce équitable (CE) sort progressivement de la confidentialité et connaît un changement d'échelle spectaculaire. Dans les associations locales de promotion du CE, relais des organisations nationales historiques telles qu'Artisans du Monde (AdM) ou Max Havelaar France (MHF), cette croissance se manifeste sous des formes variées - multiplication, diversification et spécialisation des activités, des ventes, etc. - et entraîne une forme de professionnalisation qui induit une reconfiguration plus ou moins profonde de ces dernières. À travers l'étude de deux groupes locaux - AdM et Autrement/Equi'Max - à Dijon (Bourgogne), ce texte cherche à rendre compte des multiples adaptations, notamment organisationnelles, que ces groupes connaissent avec cette phase nouvelle de croissance. De la nécessaire formalisation des instances dirigeantes à l'expérimentation de nouvelles procédures de recrutement et de formation des bénévoles face à des activités nécessitant davantage de technicité, cette contribution témoigne de la portée et des répercussions de la nouvelle visibilité du CE.
\end{abstract}

Mots clés : association ; bénévolat ; commerce équitable ; diversification ; engagement.

Thèmes : économie et développement rural ; transformation ; commercialisation.

\section{Abstract \\ Scaling up Fair Trade: an empirical reality. Local analysis of the evolution of two associations in Burgundy}

Since early 2000, French Fair Trade (FT) has been experiencing spectacular growth and is coming out of its confidential status. In local branches of national organizations promoting FT - e.g. Artisans du Monde and Max Havelaar - such growth manifests itself through a variety of forms (multiplication, diversification and specialization of work, sales, etc.) and involves a kind of professionalization that ultimately contributes to reshaping these organizations. Through carrying out a case study of two local groups Artisans du Monde and Equi'Max - in Dijon (Burgundy), the paper sheds light on the multiple organizational adaptations that these groups are experiencing in this new phase of growth. Examining the formalization of the internal organization (new boards) to the experimentation of new recruitment procedures and the training of volunteers for activities requiring more professional skills, this paper shows the scope and the repercussions of the new visibility of FT.

Key words: associations; commitment; diversification; fair trade; voluntary help.

Subjects: economy and rural development; processing, marketing. n France, depuis le début des années 2000, le commerce équitable (CE) connaît une forte croissance, en grande partie due à son implantation controversée en grandes et moyennes surfaces (GMS). À titre illustratif, le marché des produits portant le label délivré par l'association Max Havelaar France (MHF), 
disponibles dans plus de 45000 points de ventes, est passé de 5,6 millions d'euros en 2000 à 256 en 2008. Les autres acteurs associatifs historiques ne sont pas en reste : par exemple la fédération Artisans du monde (FAdM) a connu une augmentation sensible de son chiffre d'affaires entre 2000 et 2007 (de 4,4 à 10,5 millions d'euros) comme de son nombre d'antennes (de 97 à 170 points de vente). Pour parvenir à ces résultats, ces acteurs nationaux se sont lentement structurés tout en développant et parfois en impulsant une réorganisation de leurs réseaux associatifs. Ce sont en effet ces deniers, constitués de multiples antennes locales, qui promeuvent, parfois depuis une trentaine d'années, le CE dans les régions et sont donc en contact immédiat avec le public, les institutions locales, les financeurs et les autres partenaires potentiels.

Afin de mieux comprendre comment s'est opéré ce changement d'échelle, d'une pratique marginale et cantonnée à une sphère militante, à une activité en passe de s'ancrer dans les habitudes d'achat d'un nombre toujours plus important de consommateurs, nous nous sommes intéressés aux manières dont, concrètement ; au sein de plusieurs associations locales de CE, s'est produit ce changement d'échelle. Quels sont les indicateurs de la contribution des associations locales à ce changement d'échelle? Quels sont les aménagements organisationnels effectués et en quoi ont-ils un impact sur le bénévolat? De quelle nature sont les difficultés rencontrées par les associations? Telles sont quelques-unes des questions qui ont animé ce travail dont le volet empirique est issu d'un doctorat de sociologie (Gateau, 2007a) au cours duquel ont été réalisés une soixantaine d'entretiens avec des bénévoles, des dirigeants et des salariés de deux associations situées à Dijon, une des rares villes françaises comptant à la fois une antenne AdM et un groupe membre de MHF. Des entretiens complémentaires ont été menés avec des dirigeants et des salariés de la FAdM et de MHF et les archives locales et nationales de ces organisations ont été dépouillées. Enfin, l'observation de la vie de ces structures locales (assemblées générales, conseil d'administration, boutique, manifestations...) durant trois années a permis de compléter les sources de compréhension du fonctionnement de cet univers associatif.

Dans un premier temps, nous proposons une sociographie concise d'Equi'Max et d'AdM Dijon avant d'étudier les multiples voies par lesquelles les associations locales ont participé à ce changement d'échelle en tentant d'en limiter les effets pervers.

\section{Sociographie des associations locales de commerce équitable}

Depuis le milieu des années 1990, Equi'Max et AdM œuvrent à la promotion du $\mathrm{CE}$ en Bourgogne. Voici quelques éléments de présentation de ces structures.

\section{D’ " Autrement " à Equi’Max}

En 1993, à la suite d'un voyage professionnel de dix mois en Amérique du Sud, trois jeunes professionnels du milieu agricole, proches des thèses développementalistes et du mouvement coopératif agricole, ont créé une première association, "Autrement ". Il s'agissait alors de mettre en place des alternatives au développement rural inspirées des expériences coopératives découvertes à l'étranger. Très marqués, en particulier, par leur rencontre avec des caféiculteurs vénézuéliens travaillant avec MHF, alors encore embryonnaire, ces personnes souhaitaient réfléchir à la possibilité de commercialiser du café équitable en France. Après une période d'études de faisabilité de leur projet avec MHF, "Autrement", alors constituée de militants issus des réseaux relationnels et professionnels des trois fondateurs - beaucoup travaillent ou militent alors à la Chambre départementale d'Agriculture, au Centre départemental des jeunes agriculteurs (CDJA), dans l'association Agriculteurs français et développement international (ADFI) ou au Centre international de coopération pour le développement agricole (CICDA) - a commencé à promouvoir le $\mathrm{CE}$ et la certification MHF (1995). À cette fin, elle est devenue membre (conventionné) du réseau associatif développé par MHF qui, s'il n'a pas la forme d'une fédération, compte tout de même une quarantaine d'antennes locales. En 1998, "Autrement " a participé à la campagne nationale lancée par le réseau " Agir Ici " et le collectif " De l'éthique surl'étiquette ", afin d'inciter les GMS à référencer le café labellisé MHF. Couronnée de succès l'année suivante, cette campagne est, depuis et pour beaucoup d'observateurs, considérée comme à l'origine du changement d'échelle du CE.

Depuis cette époque (2000) qui a vu la demande d'information sur le CE augmenter et a conduit à la création d'un premier emploi de permanent, l'association et sa soixantaine d'adhérents organisent des animations (dégustation/sensibilisation/promotion), propose des actions d'éducation au développement (AED) auprès de publics scolaires ou professionnels et relaie diverses campagnes de lobbying initiées par MHF ou d'autres partenaires.

A la différence d'AdM, "Autrement " n'a pas de boutique et ne vend des produits équitables aux particuliers qu'à la marge, privilégiant le développement de la consommation équitable hors domicile pour laquelle un second emploi a été créé en 2005, année où elle a également été rebaptisée "Equi'Max ".

\section{AdM Dijon, membre actif de la Fédération AdM (FAdM)}

L'histoire de cette association doit beaucoup à ses fondateurs, un couple de bénévoles d'une autre antenne d'AdM venu s'installer à Dijon et qui, entre 1995 et le début des années 2000, en sera aussi le principal acteur.

Pour monter la section locale d'AdM, ce jeune couple s'appuie sur une poignée de bénévoles d'une ancienne association membre de la FAdM plusieurs années auparavant. En mobilisant diverses ressources, militantes, humaines et financières, les fondateurs, après avoir, en quelques mois, trouvé un local et constitué un stock de marchandises, sont bientôt rejoints par une petite équipe de bénévoles pour commercialiser les produits alimentaires et artisanaux provenant de la centrale d'achat de la FAdM. Entre 1995 et 2000, cette première équipe polyvalente a porté l'association et, malgré des débuts timides sur le plan commercial, elle a tenté le pari de créer un premier emploi afin d'assurer une visibilité maximale à la boutique, ouverte dès lors une trentaine d'heures par semaine. La participation des bénévoles (une quarantaine à l'époque contre une soixantaine actuellement) s'effectue depuis et pour l'essentiel au sein de la boutique (vente, gestion des stocks, information, etc.). Un nombre restreint d'entre eux gère les activités de lobbying impulsées par la FAdM, tandis que l'AED est le fait d'une petite dizaine de militants. Depuis 2005, date à laquelle 
la FAdM a entériné la décision de ne pas distribuer ses produits en GMS, une seconde salariée chargée de l'animation commerciale a été embauchée à Dijon, en particulier pour travailler au développement de partenariats commerciaux avec des entreprises et institutions locales.

En une quinzaine d'années, cette " petite " association est devenue un membre important, tant sur le plan commercial qu'en termes de participation à la politique associative du réseau, parmi les 160 associations que compte la FAdM, puisque plusieurs militants dijonnais siègent ou ont siégé à la FAdM (présidence, conseil d'administration, commissions de travail...).

Après cette présentation sommaire, venons-en au changement d'échelle du CE et à la manière dont il s'est produit dans les associations locales.

\section{Accompagner}

\section{le changement} d'échelle du CE : une réalité locale aux multiples facettes

À partir du début des années 2000, la médiatisation du CE permise par son entrée dans les GMS participe d'une croissance qui, depuis, ne se dément pas et concerne l'ensemble des acteurs et des filières, qu'elles soient spécialisées pour la FAdM ou certifiées avec MHF.

Dans les associations locales, le succès est explicite : sur la période 2000-2007, où cette croissance est la plus forte, le chiffre d'affaires d'AdM Dijon est passé de 106000 euros à près de 200 000. Dans le même temps, les sollicitations se sont diversifiées et n'ont cessé de croître, surtout en matière d'AED où les animations ont été triplées, passant de 34 à plus de 100 par an. Les effectifs se sont eux graduellement étoffés pour parvenir à une centaine de membres. Pour Equi'Max, le constat est similaire : si les effectifs se sont stabilisés autour d'une soixantaine d'adhérents, le nombre d'animations a plus que doublé entre 1999 et 2006 (de 20 à 49, permettant de dispenser une information sur la certification et le CE à plus de
7500 personnes par an) tandis que les sources de financements se sont élargies. Au-delà d'une hausse quantitative des activités, une hausse qualitative, résultant de l'embauche de permanents salariés, de la professionnalisation des bénévoles (formation) ou encore de la mise en place de procédures de recrutement et de suivi des bénévoles, a été observée. Celle-ci traduit une volonté de sortir de l'amateurisme originel, en partie impulsée par les organisations nationales de $\mathrm{CE}$, et concomitante d'une technicisation de certaines activités qui, après avoir, plusieurs années durant, fait l'objet d'un " bricolage " artisanal, sont désormais davantage normées et encadrées. Ces transformations n'ont pu se produire que par un travail de fonds des associations et de leurs bénévoles qui les ont conduites à une série de reconfigurations internes dont les résultats apparaissent mitigés.

\section{Le développement ou la nécessaire adaptation des associations}

Comme nous l'avons souligné, les deux associations locales, si elles sont actrices du changement d'échelle du CE, ont également dû s'y adapter, au prix d'une réorganisation interne polymorphe. Bien plus qu'une spécificité due à l'histoire locale de tel ou tel collectif, ces évolutions concernent l'ensemble des acteurs associatifs, locaux comme nationaux, qui ont dû faire face à une démocratisation massive de "l'objet " CE.

Pour Equi'Max, historiquement fondée sur un réseau d'interconnaissances constitué autour de professionnels et de militants de la sphère agricole locale, la première reconfiguration d'envergure a consisté en la formalisation de ses instances dirigeantes. En effet, durant ses premières années, l'association a fonctionné de manière (très) conviviale et informelle, sans bureau, dans un idéal de démocratie directe sans hiérarchisation (Ion et al., 2001) qui faisait dire à ses fondateurs qu' "Autrement " était "une association de copains". Cependant, en raison de la multiplication, de la diversification et de la technicisation progressive des tâches accompagnant la croissance du CE, l'association a été confrontée à de multiples difficultés de coordination, de fonctionnement et de définition de ses projets (nature des liens avec MHF, division du travail, des responsabilités, problèmes de recrutement et de formation des béné- voles, etc.) qui a conduit au remaniement de son organisation interne. Un bureau a été élu ainsi que des référents par domaine d'activité, afin de gagner en efficacité en spécialisant les différents secteurs d'intervention du collectif et en mettant en place un suivi des activités et des bénévoles. Mais cela n'a pas suffi à résoudre la totalité des problèmes, d'autant que les fondateurs, entraînant dans leur sillage de nombreux militants de la première heure, ont quitté le groupe au début des années 2000. Pour eux, cette formalisation a nui à la convivialité et à l'esprit originel du groupe, tandis que les "dérives " commerciales et marketing du système MHF - au détriment de questions plus politiques concernant l'avenir de l'agriculture et le sort des producteurs des pays du Sud - n'étaient plus compatibles avec leur vision du CE et la vocation initiale d'un collectif de moins en moins uni. C'est d'ailleurs dans ce contexte délicat, à la fois pour clarifier ses liens avec MHF, mais aussi pour tourner une page de son histoire, que le nom de l'association a été modifié en 2005.

AdM a connu une adaptation différente que l'on peut restituer à travers diverses séquences. C'est en effet à partir de l'année 1999 que l'association connait ses transformations les plus importantes, d'abord avec le départ ou plus exactement la mise en retrait des fondateurs et plus spécifiquement de celle qui en a été la présidente jusqu'à cette date. Désireuse de "passer la main " tout en entamant une carrière militante nationale qui la conduira à être élue vice-présidente (1999) puis présidente de la FAdM (2004-2007), cette dernière a marqué de son empreinte le collectif local. À cet égard, aucun candidat n'a souhaité lui succéder à la tête de l'association dijonnaise et, depuis, AdM Dijon fonctionne sans président, mais avec un conseil d'administration. Cependant, après quelques difficultés de fonctionnement et face au scepticisme grandissant de certains adhérents vis-à-vis de cette organisation collégiale, mais aussi pour faire face à la hausse des sollicitations (commerciales, AED, conférences, création de contenus pédagogiques...), des commissions de travail ont été créées afin de rationaliser et mieux structurer les pratiques et l'organisation internes. En 2000, la relocalisation de l'association aux portes du centre-ville a constitué un autre événement décisif, puisqu'un an plus tard, le chiffre d'affaires de l'association avait augmenté de $36 \%$ et le nombre de bénévoles avait doublé (de 40 à 80 ). 
Dans les deux collectifs, la poursuite de la salarisation, débutée en 1995 pour AdM et en 1999 pour Equi'Max, constitue un autre indicateur de ce changement d'échelle, avec la création d'un second emploi subventionné dédié à l'AED dans chacune des associations (2005). Ce processus de salarisation est essentiel pour comprendre à la fois le besoin de structuration et de suivi qui est apparu dans ces collectifs (gestion du "personnel ", coordination, approvisionnement commercial...), mais aussi pour bien saisir la manière dont les associations ont souhaité faire face à une technicisation de certaines activités. À ce propos, on peut noter très concrètement des évolutions notables dans tous les domaines d'activités pour lesquels la "bonne volonté " ne suffit plus et doit être accompagnée de certaines compétences et savoir-faire spécifiques : maîtrise des outils informatiques (comptabilité, gestion des approvisionnements et stocks de marchandises, des achats...), aptitudes oratoires (interventions en public), connaissances économiques et pédagogiques (AED), capacités à monter des dossiers de subventionnement, à démarcher des partenaires commerciaux habitués à traiter avec des professionnels, etc. Ainsi, et plus encore avec le changement d'échelle évoqué, il apparaît que les pratiques d'amateurs des débuts ne correspondent plus ni aux besoins actuels des associations, ni aux exigences toujours plus nombreuses des clients et des financeurs, ni aux... impératifs commerciaux. Chez AdM, cette émancipation de l'amateurisme a pris une tournure encore plus visible en 2008, lorsqu'a été adoptée la charte graphique nouvellement instaurée par la FAdM afin d'harmoniser les boutiques locales (mobilier, signalétique, couleurs, packaging...) pour augmenter leur visibilité et leur donner une allure plus "professionnelle ", à l'image des franchises du secteur commercial traditionnel.

À l'heure où l'univers du CE est traversé par ce que certains nomment une "crise de croissance "(Courrier de la Planète, 2009), nombreux sont les acteurs qui souhaitent offrir une garantie de sérieux et de professionnalisme tout en cherchant à rassurer des consommateurs perturbés par le foisonnement des pseudo-labels et autres certifications, qui nuisent à la crédibilité même du CE... et remettent à l'ordre du jour certaines questions fondamentales (garantie, norme, transparence, etc.) qui agitent le secteur depuis longtemps mais restent sans réponse.

\section{Mieux recruter, former et retenir les bénévoles}

En matière de mobilisation, de renouvellement et de formation des bénévoles, nous avons montré ailleurs (Gateau, 2007b) que diverses stratégies ont été mises en place par les associations investiguées pour gérer au mieux les transformations évoquées, parfois dans le cadre d'une politique d'accompagnement et de structuration des réseaux associatifs mise en place par la FAdM ou MHF. Voici, de manière très synthétique, quelques-unes de ces mesures :

- diversification des lieux et modalités de recrutement (tracts, annonces, centre du volontariat, réseaux professionnels...) et dépassement de la cooptation ;

- travail d'identification des compétences, savoir-faire, envies et motivations des bénévoles, désormais invités à passer un " entretien d'embauche "à leur entrée dans les associations ;

- systématisation des formations proposées aux nouveaux entrants, parfois organisées en partenariat avec la FAdM ou MHF: pour "rendre le travail des bénévoles plus attrayant, pour renforcer leur motivation"; pour "élargir leurs compétences et mieux répondre à la demande (...) le recours à une formation est indispensable" (Halba, 2003);

- fidélisation des bénévoles ;

- ouverture à de nouveaux publics.

Sur ce dernier point, il s'agit en particulier de contrer le vieillissement généralisé des effectifs. À titre d'exemple, en 2006, 60 \% des adhérents d'AdM Dijon avaient plus de 50 ans et seulement $7 \%$ moins de 30 ans. Cette caractéristique, qui renvoie directement à l'allongement de la durée de la vie et à la participation croissante des seniors à la sphère associative et bénévole (Michaudon, 2002), permet de comprendre pourquoi les associations cherchent à attirer des jeunes et en particulier à nouer des partenariats avec le monde estudiantin, très prisé, même s'il représente un public traditionnellement volatil...

Ces pistes de réflexion et d'expérimentation, non exhaustives, induisent des contraintes à la fois pour les nouveaux adhérents et pour les associations, et modifient nécessairement l'organisation de ces dernières, qui se formalise. En plus de chercher à mieux former et recruter une "main-d'œuvre qualifiée ", ces mesures s'inscrivent dans une réflexion récurrente sur les moyens à mettre en œuvre ou à inventer pour parvenir à fidéliser les bénévoles. Il s'agit également, in fine, de lutter contre un fort turn-over qui, s'il n'est pas un phénomène local, constitue "l'un des traits permanents des organisations politiques, qu'elles soient partisanes, syndicales ou associatives" (Filleule et al., 2005). Or, en la matière, aucune piste sérieuse ne semble avoir encore été trouvée...

\section{Conclusion}

À travers les éléments évoqués, qui sont autant d'indicateurs du changement d'échelle du CE au niveau local, nous avons souhaité montrer que les associations de CE travaillent continûment depuis près d'une quinzaine d'années pour s'adapter et accompagner cette croissance qui transforme progressivement non seulement leur visage, mais aussi les manières qu'on a d'y pratiquer le bénévolat. En effet, au-delà même de la cause défendue, militer dans une association fondée sur l'interconnaissance et un fonctionnement quasi informel et amateur n'a pas la même signification que faire partie d'une organisation dans laquelle l'efficacité, la rentabilité, la compétence et, in fine, le professionnalisme deviennent les maîtres mots. Impliquant assiduité, formation(s), expérience, voire prise de responsabilités, cette rationalisation des activités apparaît difficilement compatible avec l'amateurisme, la "débrouille " et la norme de non-contrainte, aux sources du bénévolat tel qu'il est traditionnellement pensé. Du fait de ces évolutions, certains des bénévoles les plus anciens affirment regretter la période précédant le changement d'échelle du CE. Si les activités associatives étaient peut-être moins nombreuses et les résultats commerciaux plus faibles, les liens entre les adhérents, davantage tournés vers le volet politique du CE et son projet de transformation social, semblaient plus forts et d'une autre nature.

Finalement, si le changement d'échelle du CE est bien synonyme d'une croissance, laquelle se traduit notamment par des résultats financiers en hausse, il induit également son lot d'effets pervers qui conduisent à en contraster le bilan. En effet, lorsque les collectifs d'appartenance se technicisent, au point d'adopter certaines normes issues du monde de l'entreprise et de devenir des "entreprises associatives" 
(Hély, 2004), le sens de l'engagement bénévole se transforme et n'intéresse pas ou plus ceux qui viennent y effectuer un "travail" souvent de plus en plus éloigné du plaisir de donner et de faire partie d'une association. Cela engendre donc des écueils en matière de participation, de renouvellement et de fidélisation des bénévoles, avec comme conséquence de favoriser des engagements ponctuels au détriment du développement, à moyen ou long terme, d'un véritable projet collectif et militant. Cela signifie, à l'encontre d'une vision stéréotypée de l'engagement, que nombreux sont les adhérents à ne pas ou ne plus être prêts à se donner " corps et âme "à leur organisation et à restreindre par conséquent leur engagement à certaines sphères temporelles et domaines d'activités, surtout si ceux-ci sont potentiellement pourvoyeurs de satis- factions, voire de rétributions symboliques (Gaxie, 1977). Partant de là, et face à la place toujours plus importante accordée aux salariés, la question du rôle, du renouvellement interne et même de la pérennisation de ces petites associations locales, reste plus que jamais d'actualité.

\section{Références}

Commerce équitable: la crise de croissance ? Courrier de la Planète 2009 ; 87 : 1-70.

Filleule O, Broqua C, Gottraux P. Le désengagement militant. Paris : Belin, 2005.

Gateau M. Les militants du commerce équitable. Analyse localisée de l'engagement dans deux associations. Dijon: université de Bourgogne, 2007a.
Gateau M. Du bouche-à-oreille à l'entretien "d'embauche". Le recrutement dans les associations de commerce équitable. Sociologies Pratiques, 2007b; 15: 123-34. doi: $10.3917 /$ sopr.015.0123

Gaxie D. Économie des partis et rétribution du militantisme. Revue française de sciences politiques $1977 ; 1: 123-54$.

Halba B. Bénévolat et volontariat en France et dans le monde. Paris: La Documentation Française, 2003.

Hély M. Les différentes formes d'entreprises associatives. In Ferrand-Bechmann D, ed. Les bénévoles et leurs associations. Autres réalités, autre sociologie? Paris: L'Harmattan, 2004.

Ion J, Belbahri A, Charvolin $\mathrm{F}$, et al. L'engagement au pluriel. Saint-Etienne: PUSE, 2001.

Michaudon H. L'engagement associatif après 60 ans. Paris : INSEE Première, 2002. 